\title{
Lactate and number of organ failures predict intensive care unit mortality in patients with acute-on-chronic liver failure
}

\author{
Filipe S. Cardoso ${ }^{1}$ (D) | Juan G. Abraldes ${ }^{2}$ (D) | Eric Sy ${ }^{3}$ | Juan J. Ronco ${ }^{3}$ | Luís Bagulho ${ }^{4}$ | \\ Mark J. Mcphail ${ }^{5}$ (D) | Constantine J. Karvellas ${ }^{2,6}$
}

\author{
${ }^{1}$ Gastroenterology and Intensive Care \\ Divisions, Central Lisbon Hospital \\ Center, Curry Cabral Hospital, Lisbon, \\ Portugal \\ ${ }^{2}$ Gastroenterology Division (Liver \\ Unit), University of Alberta Hospital, \\ Edmonton, AB, Canada \\ ${ }^{3}$ Critical Care Division, Vancouver General \\ Hospital, Vancouver, BC, Canada \\ ${ }^{4}$ Intensive Care Division, Central Lisbon \\ Hospital Center, Curry Cabral Hospital, \\ Lisbon, Portugal \\ ${ }^{5}$ Liver Intensive Therapy Unit, King's College \\ Hospital, London, UK \\ ${ }^{6}$ Critical Care Department, University of \\ Alberta Hospital, Edmonton, AB, Canada

\section{Correspondence} \\ Filipe S. Cardoso, Intensive Care Unit, \\ Central Lisbon Hospital Center, Curry Cabral \\ Hospital, Lisbon, Portugal. \\ Email: filipe_sousacardoso@hotmail.com
}

Handling Editor: Dominique Thabut

\author{
Abstract \\ Background and Aims: Patients with acute-on-chronic liver failure (ACLF) have high \\ mortality rates. Most prognostic scores were not developed for the intensive care \\ unit (ICU) setting. We aimed to improve risk stratification for patients with ACLF in \\ the ICU.
}

Methods: A training set with 240 patients with cirrhosis and organ failures (Chronic Liver Failure Sequential Organ Failure Assessment score [CLIF-SOFA]) from Curry Cabral Hospital (Portugal) and University of Alberta Hospital (Canada) in 2010-2016 was used to derive a prognostic model for ICU mortality. A validation set with 237 patients with cirrhosis and organ failures from Vancouver General Hospital (Canada) in 2000-2011 was used to evaluate its performance.

Results: Amongst patients in the training set, ICU and hospital mortality rates were $39.2 \%$ and $54.6 \%$ respectively. Median lactate ( $4.4 \mathrm{vs} 2.5 \mathrm{mmol} / \mathrm{L}$ ) and number of organ failures ( 3 vs 2 ) on admission to ICU were associated with higher likelihood of ICU mortality $(P<0.001$ for both). The lactate and organ failures predictive model (LacOF) was derived to predict ICU mortality: $-2.420+0.072 \times$ lactate $+0.569 \times$ number of organ failures (area under-the-curve [AUC], 0.76). In the validation set, the LacOF model discriminative ability (AUC, 0.85) outperformed the CLIF-SOFA (AUC, 0.79), Chronic Liver Failure Consortium Acute-on-Chronic Liver Failure (AUC, 0.73), Model for End-stage Liver Disease score (AUC, 0.78) and Acute Physiology and Chronic Health Evaluation II scores (AUC, 0.74; $P<0.05$ for all). The LacOF model calibration was good up to the $25 \%$ likelihood of ICU mortality.

Conclusions: In patients with ACLF, lactate and number of organ failures on admission to ICU are useful to predict ICU mortality. This early prognostic evaluation may help to better stratify the risk of ICU mortality and thus optimize organ support strategies.

KEYWORDS

cirrhosis, critical care, death, prognosis 


\section{1 | INTRODUCTION}

Patients with cirrhosis and organ failures have been defined as having acute-on-chronic liver failure (ACLF). ${ }^{1}$ This clinical entity portends significantly higher overall mortality than acutely decompensated cirrhosis. ${ }^{2}$ Despite the nature of the precipitant event associated with the acute deterioration (eg infection, bleeding or other), the evolving systemic inflammatory response syndrome (SIRS) eventually leads to organ failures. As organ failures add, the overall mortality increases. ${ }^{3}$

Patients with ACLF admitted to the intensive care unit (ICU) have frequently a bad short-term prognosis and may be deemed too sick for non-futile liver transplant (LT). Although most rules of care for general critically ill patients apply, there are specific features of these patients that often create additional challenges to their acute management and may worsen their prognosis, for example the increased susceptibility to infection and complications of portal hypertension such as refractory ascites, variceal bleeding, hepatic hydrothorax or hepatorenal syndrome. Nevertheless, even if the LT window of opportunity may be difficult to ascertain, organ support strategies need to be timely initiated in order to maximize the likelihood of survival. ${ }^{4,5}$

Existing prognostic tools for patients with cirrhosis not necessarily admitted to the ICU (eg Chronic Liver Failure-C ACLF [CLIF-C ACLF] and Model for End-stage Liver Disease [MELD] scores) or for the general critically ill patients (eg Sequential Organ Failure Assessment [SOFA] and Acute Physiology and Chronic Health Evaluation II [APACHEII] scores) have been applied to patients with ACLF in the ICU to stratify these patients short-term likelihood of morbidity and/or mortality. ${ }^{6,7}$ However, as they have not been developed specifically to prognosticate ICU-related outcomes for patients with ACLF, there may be limitations to their use in this context.

Taking this into account, we aimed to assess the performance of current prognostic systems for patients with ACLF while in the ICU. Furthermore, we sought to develop an alternative prognostic tool for this specific setting.

\section{2 | MATERIALS AND METHODS}

\section{1 | Study design, participants and data collection}

This was an international multicentre retrospective cohort study. A training set included consecutive patients with cirrhosis and organ failures admitted to the ICU of two LT centres: Curry Cabral Hospital (Lisbon, Portugal) from 1 April 2013 to 31 December 2016 ( $n=70$ ); and University of Alberta Hospital (Edmonton, Canada) from 1 March 2010 to 30 September 2013 ( $n=170$ ). A validation set included consecutive patients with cirrhosis and organ failures admitted to the ICU of another LT centre: Vancouver General Hospital (Vancouver, Canada) from 1 June 2000 to 31 March 2011 ( $=237$ ).

Patients were included if fulfilling the following criteria: age $\geq 18$ years; diagnosed with cirrhosis and organ failures (defined by Chronic Liver Failure Sequential Organ Failure Assessment

\section{Key points}

- Patients with cirrhosis may develop several organ failures (acute-on-chronic liver failure) and become so sick they require admission to intensive care unit (ICU).

- As previous prognostic tools have not been created specifically for the ICU setting, we developed a new prognostic tool to estimate their probability of surviving the ICU stay based on two clinical parameters measured as early as on admission to ICU: lactate $(\mathrm{mmol} / \mathrm{L})$ and the number of organ failures.

- This risk score was validated against other pre-existing prognostic tools and showed an overall good performance.

[CLIF-SOFA] score); and on first admission to ICU during the inclusion period at each study site. Exclusion criteria were: diagnosis of acute liver failure; diagnosis of decompensated cirrhosis without organ failures; or previous LT.

All data on patients' characteristics were retrieved from manual and electronic medical records and collected in an anonymous and protected database. The following data were retrieved on admission to ICU: age, sex, aetiology and complications (ascites and hepatocellular carcinoma) of cirrhosis, precipitant event of acute illness, number and severity of organ failures and their level of support, general laboratory parameters and severity of illness systems (see Definitions, exposures and endpoints3.2). In all patients, lactate was measured in the first arterial blood withdrawn from the arterial line inserted immediately following admission to the ICU.

As this was a non-interventional study, local ethics committees waived the need for individual informed consent. All study procedures followed the principles of the Declaration of Helsinki. ${ }^{8}$ The reporting of this study followed the Strengthening the Reporting of Observational Studies in Epidemiology (STROBE) guideline. ${ }^{9}$

\section{2 | Definitions, exposures and endpoints}

Cirrhosis was defined as a bridging fibrosis on previous liver biopsy or a composite of clinical signs and findings provided by laboratory tests, endoscopy and radiologic imaging. ${ }^{1}$ Organ failures and ACLF criteria were defined based on the CLIF-SOFA score as per European Foundation for the Study of Chronic Liver Failure (CLIF) Consortium. ${ }^{2}$

The ACLF grading system ranks patients with ACLF in one of three grades based on the number of organ failures according to the CLIF-SOFA score: grade 1 if (a) single kidney failure (serum creatinine of $\geq 2.0 \mathrm{mg} / \mathrm{dL}$ ) is present, (b) another organ failure (liver, coagulation, circulation or respiration) is present with a serum creatinine of 1.5-1.9 $\mathrm{mg} / \mathrm{dL}$ and/or there is grade I-II (West Haven criteria) hepatic encephalopathy (HE) or (c) single cerebral failure (grade III-IV HE) is 
present with a serum creatinine of $1.5-1.9 \mathrm{mg} / \mathrm{dL}$; grade 2 if 2 organ failures are diagnosed; or grade 3 if 3 or more organ failures have developed. ${ }^{2}$

Severity of illness systems considered on admission to ICU was: CLIF-SOFA, CLIF-C ACLF, CLIF-C ACLF Lac, APACHEII and MELD scores. The CLIF-SOFA score is a validated adaptation of the classical SOFA to assess the severity of disease in patients with cirrhosis derived by the CLIF Consortium. It comprises the grading of six different systems of organs (ranging each from 0 [least severe] to 4 [most severe] and overall from 0 to 24): brain, cardiovascular system, lungs, kidneys, liver and coagulation. ${ }^{2}$ The CLIF-C ACLF score prognosticates 28-day mortality in patients with ACLF by adding the number of organ failures, defined by the CLIF-SOFA score, to age and white blood cells' count. ${ }^{7}$ The CLIF-C ACLF Lac score was recently developed to prognosticate 28-day mortality in patients with cirrhosis in the ICU by adding lactate level on admission to ICU to the CLIF-C ACLF score. ${ }^{10}$ The APACHEII score has been widely used for several decades to assess the severity of disease for general patients in the ICU. It is based on 12 routine physiologic measurements, age, previous health status (chronic disease and/or immunodeficiency) and surgical status to predict the likelihood of hospital mortality (ranging overall from 0 to 71). ${ }^{11}$ The MELD score has been initially developed to predict 3-month mortality for patients with cirrhosis and portal hypertension who underwent a transjugular intrahepatic portal shunt procedure but is now widely used to assess their likelihood of 3-month mortality while in the waitlist for LT. ${ }^{12}$ It is based on the evaluation of liver (INR and bilirubin) and renal (creatinine) functions. Both in Portugal and Canada, candidates for LT are prioritized based on the MELD score.

The primary endpoint considered was all-cause mortality within the index ICU stay (irrespective of LT status). We chose this primary outcome as it may better reflect the ICU management provided to patients and because ICU mortality represented $65.2 \%$ of all deaths that occurred within 6-months following admission to ICU in the training set. Furthermore, the sensitivity analysis performed, excluding patients who were transplanted during the follow-up, did not show any impact of LT status in this study of the associations with ICU mortality. The secondary endpoints defined were all-cause mortality within the index hospital stay (irrespective of LT status) and the length of index ICU and hospital stays.

\section{3 | Statistical analysis}

Continuous and categorical variables were initially described as median (interquartile range [IQR]) and frequency (percentage [\%]) respectively. Univariate comparisons were done using Mann-Whitney and chi-square tests where appropriate. Multivariate analysis was performed with logistic regression. Survival analysis used KaplanMeier curves with comparisons being done with the Breslow test. Overall missing data on admission to ICU were $0.5 \%$.

The development of the new predictive model (Lisbon and Edmonton) initially included variables deemed clinically significant and/or with a $P<0.10$ on the univariate analysis. Its further development used a backward stepwise elimination process with the final model being the one yielding the best fit. Collinearity was avoided where appropriate. Model's performance was evaluated as per most recent statistical recommendations: discriminative ability (ie the ranking of patients based on the likelihood of ICU mortality) by area under the receiver-operating curve (AUC), with the comparisons between AUCs being done with the De Long test; calibration (ie the ability to predict an absolute likelihood of ICU mortality based on how closely the actual and predicted outcomes agree) by plotting observed against predicted ICU mortality rates; and overall performance with $R^{2}$ and Brier score (higher $R^{2}$ and lower Brier score indicate better performance). ${ }^{13}$ Internal validation was done using bootstrapping (1000 samples). External validation was performed using another cohort from a different region (Vancouver).

Statistical significance was defined as $P<0.05$ (two-tailed). Statistical analysis was performed using IBM SPSS Statistics, version 20 (IBM Corp, North Castle, NY), MedCalc Statistical Software, version 16.4.3 (MedCalc Software bvba, Ostend, Belgium) and R, with rms and predictABEL packages (R Foundation for Statistical Computing, Vienna, Austria).

\section{3 | RESULTS}

\subsection{Baseline characteristics and outcomes for the training set}

A total of 240 patients (70 from Curry Cabral Hospital and 170 from University of Alberta Hospital) fulfilled inclusion criteria. All characteristics on admission to ICU for the training set are depicted in Table 1.

On admission to ICU, the median (IQR) number of organ failures (based on CLIF-SOFA score) was 3 (2-4) with the following distribution: liver failure in 61 (25.4\%) patients; renal failure in 100 (41.7\%) patients; cerebral failure in 155 (64.6\%) patients; coagulation failure in 67 (27.9\%) patients; shock in 156 (65.0\%) patients; and respiratory failure in 105 (43.8\%) patients. The most frequent precipitant events of ACLF were sepsis and gastro-intestinal bleeding, in 82 (34.2\%) (54 of those with shock) and 32 (13.3\%) (19 of those with shock) patients respectively.

During the index ICU and hospital stays, 94 (39.2\%) and 131 (54.6\%) patients died respectively. Overall cumulative survival is displayed in Figure S1. Fifteen (6.3\%) patients were transplanted during the follow-up and four of these died within the index hospital stay (Table S1). Median (IQR) length of ICU and hospital stays were 6 (3-11) and 19 (9-34) days respectively. Median (IQR) length of follow-up was 33 (8-508) days.

In the training set, the baseline characteristics significantly associated with higher likelihood of ICU mortality (unadjusted analysis) were the following: age (59 vs 56 years), platelets ( 63 vs $88 \times 10^{3}$ ) $\mu \mathrm{L}$ ), INR ( 2.3 vs 1.7 ), bilirubin ( 7.3 vs $3.6 \mathrm{mg} / \mathrm{dL}$ ), lactate ( 4.4 vs $2.5 \mathrm{mmol} / \mathrm{L}$ ), number of organ failures ( 3 vs 2 ), need for renal replacement therapy (30.9\% vs $17.8 \%)$, need for vasopressors $(73.4 \%$ 
TABLE 1 Baseline characteristics stratified by mortality within the ICU stay in the training set

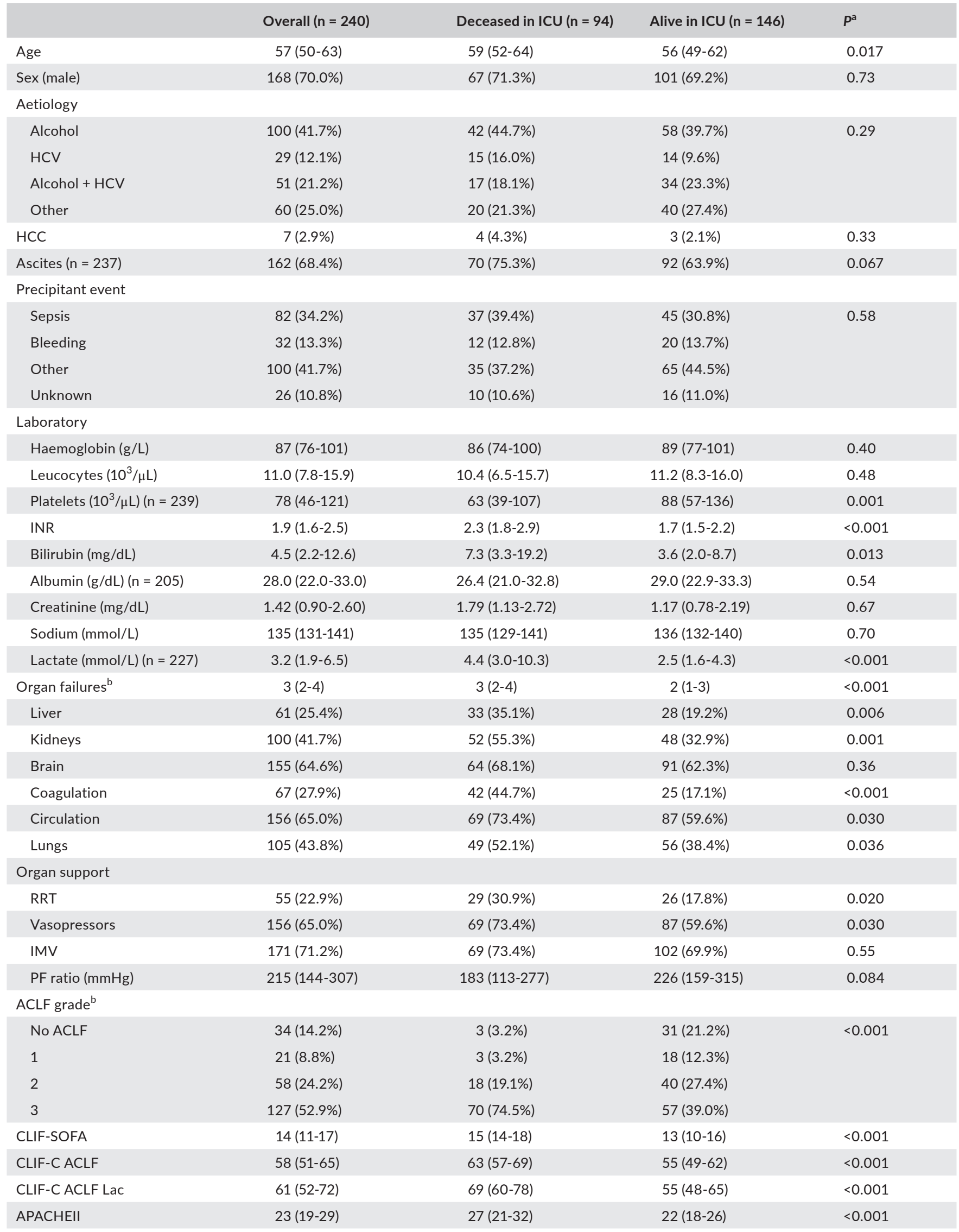


TA B L E 1 (Continued)

$\begin{array}{lcccc} & \text { Overall }(\mathrm{n}=240) & \text { Deceased in ICU }(\mathrm{n}=94) & \text { Alive in ICU }(\mathrm{n}=146) & P^{\mathrm{a}} \\ \text { MELD } & 27(17-34) & 30(24-38) & 22(15-32)\end{array}$

ICU, intensive care unit; HCV, hepatitis C virus; HCC, hepatocellular carcinoma; INR, international normalized ratio; RRT, renal replacement therapy; IMV, tracheal intubation with or without invasive mechanical ventilation; PF ratio, arterial oxygen partial pressure/oxygen inspiration fraction ratio; ACLF, acute-on-chronic liver failure; CLIF-SOFA, Chronic Liver Failure - Sequential Organ Failure Assessment score; CLIF-C ACLF, Chronic Liver Failure - Acute-on-Chronic Liver Failure score; CLIF-C ACLF Lac, Chronic Liver Failure - Acute-on-Chronic Liver Failure - Lactate score; APACHEII, Acute Physiology and Chronic Health Evaluation II score; MELD, Model for End-stage Liver Disease score (biochemical).

anivariate analysis with logistic regression $(\alpha=0.05)$.

${ }^{b}$ Definitions of organ failures based on CLIF-SOFA score.

vs $59.6 \%)$, ACLF grading system (74.5\% vs $39.0 \%$ for grade 3 ), and CLIF-SOFA (16 vs 13), CLIF-C ACLF (63 vs 55), CLIF-C ACLF Lac (69 vs 55), APACHEII (29 vs 23) and MELD scores (30 vs 22$)(P<0.04$ for all). The unadjusted association of lactate and the number of organ failures with ICU mortality is presented in more detail in Figures S2 and S3 respectively. Cumulative survival stratified by lactate and the number of organ failures on admission to ICU is displayed in Figure S1.

Median lactate (3.0 vs 2.0) and the number of organ failures ( 3 vs 2 ) on day 3 following admission to ICU were also associated with ICU mortality in the unadjusted analysis ( $P<0.001$ for both) but not in the adjusted analysis (Table S2), therefore we decided to use data on lactate and the number of organ failures from admission to ICU for the predictive analysis. ${ }^{10}$

\subsection{Development of the new predictive model}

To start developing the new predictive model for ICU mortality, we initially included the following variables: age, sex, ascites, platelets, INR, bilirubin, lactate, number of organ failures, need for renal replacement therapy, need for vasopressors and arterial oxygen partial pressure/oxygen inspiration fraction ratio. Through a backward stepwise elimination process, the best predictive model for ICU mortality derived comprised the following variables (Table 2): lactate (adjusted odds ratio $[\mathrm{aOR}]=1.08$ ) and the number of organ failures $(a O R=1.77)(P<0.04$ for both). The new predictive model (lactate and organ failures predictive model [LacOF]) performed reasonably well in the training set with an AUC of 0.76 , a $R^{2}$ of 0.23 and a Brier score of 0.20 (Figure 1A). Overall, $68 \%$ of the patients were correctly classified. The logistic regression equation derived was the following: $-2.420+0.072 \times$ lactate $+0.569 \times$ number of organ failures (with lactate in $\mathrm{mmol} / \mathrm{L}$ and number of organ failures ranging from 1 to 6 according to the CLIF-SOFA score). An estimation of the individual probability of ICU mortality based on lactate ( $\geq 3$ vs $<3$ - a cutoff defined based on the observed median value and best balance of sensitivity [78\%] and specificity [60\%]) and the number of organ failures on admission to ICU is displayed in Figure 2.

In the training set (Table 3 and Figure S4), the discriminative ability for ICU mortality of the LacOF model was significantly better than the CLIF-SOFA score $(P<0.05)$, but similar to the CLIF-C ACLF, CLIF-C ACLF Lac, APACHEII and MELD scores ( $P>0.05$ for all). However, the APACHEll overestimated the likelihood of ICU mortality for almost
TAB LE 2 LacOF model to predict mortality within intensive care unit stay

\begin{tabular}{|llll} 
& OR $(95 \% \mathrm{Cl})$ & $\mathrm{aOR}(95 \% \mathrm{Cl})^{\mathrm{a}}$ & $\mathrm{P}^{\mathrm{b}}$ \\
\hline Lactate $(\mathrm{mmol} / \mathrm{L})$ & $1.14(1.07-1.21)$ & $\begin{array}{l}1.08 \\
(1.01-1.15)\end{array}$ & 0.035 \\
& & 1.77 & 0.001 \\
\hline $\begin{array}{c}\text { Number of organ } \\
\text { failures }^{c}\end{array}$ & $1.94(1.53-2.45)$ & $(1.36-2.29)$ & \\
\hline
\end{tabular}

$y=-2.420+0.072 \times$ lactate $+0.569 \times$ number of organ failures. Probability of mortality within intensive care unit stay $=e^{y} /\left(e^{y}+1\right)$.

$\mathrm{OR}$, odds ratio; aOR, adjusted odds ratio; $\mathrm{Cl}$, confidence interval; $\mathrm{AUC}$, area under the receiver-operating characteristic curve.

aLacOF model: 89/227 patients deceased within the intensive care unit stay; AUC (95\% CI), 0.76 (0.70-0.82); Nagelkerke $R^{2}, 0.23$.

${ }^{b}$ Multivariate analysis with logistic regression after bootstrapping (1000 samples) $(\alpha=0.05)$.

${ }^{c}$ Definitions of organ failures based on Chronic Liver Failure - Sequential Organ Failure Assessment score (ranging from 1 to 6).

the entire probability scale (Figure $1 \mathrm{~B}: R^{2}$ of -0.07 and a Brier score of 0.23). Such APACHEII miscalibration in the training set further emphasized the need to develop a new predictive model for ICU mortality in patients with cirrhosis and organ failures.

To account for the possible effect of LT in the association of lactate and the number of organ failures on admission to ICU with ICU mortality, we performed a sensitivity analysis excluding the 15 patients that were transplanted during the follow-up (Table S3). This analysis yielded similar results to the original one with an $\mathrm{aOR}=1.07$ for lactate and $\mathrm{aOR}=1.74$ for the number of organ failures ( $P<0.04$ for both).

\section{3 | External validation of the new predictive model (validation set)}

A total of 237 patients from Vancouver General Hospital fulfilled inclusion criteria. All characteristics on admission to ICU for the validation set are depicted in Table 4. In comparison to the training set, the validation set had significantly higher median lactate (4.7 vs $3.2 \mathrm{mmol} / \mathrm{L} ; P<0.001$ ) but similar median number of organ failures ( 2 vs $3 ; P=0.25$ ). Furthermore, the two sets had similar proportion of ACLF grade 3 (46.8\% vs $52.9 \%)$ and median CLIFSOFA (14 vs 14), CLIF-C ACLF (58 vs 58), CLIF-C ACLF Lac (61 vs 62), APACHEII (23 vs 23; $P=0.77$ ) and MELD scores (27 vs 27; $P>0.05$ for all). 
(A) $\operatorname{tLacOF}(P=0.89)$

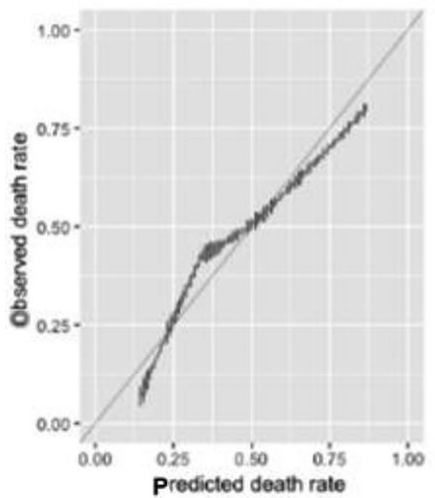

(B) TAPACHEII $(P=0.007)$

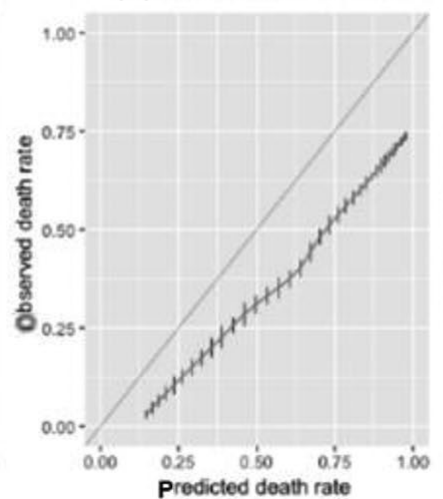

(C)

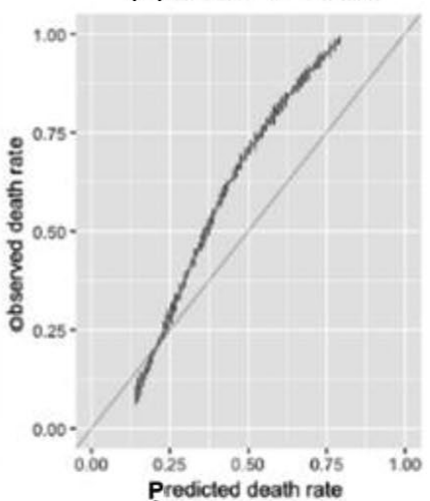

(D) VAPACHEII $(P=0.98)$

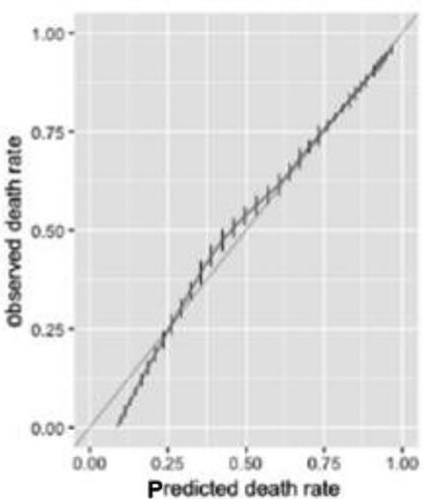

FIGURE 1 Calibration plots for lactate and organ failures predictive model (LacOF) and Acute Physiology and Chronic Health Evaluation II score (APACHEII) score in the training (A: tLacOF and B: tAPACHEII) and validation (C: vLacOF and D: vAPACHEII) sets. To develop these plots, the sample was split into quintiles and actual mortality ( $y$ axis) was plotted against predicted mortality ( $x$ axis). Points below and above the diagonal line (perfect prediction) indicate overestimation and underestimation of mortality respectively. $P$ values derive from the Hosmer-Lemeshow goodness-of-fit test (the lower the $P$ value, the worse agreement between actual and predicted mortalities)

In the validation set, during the index ICU and hospital stays, 121 (51.1\%) and 151 (63.7\%) patients died respectively. These ICU and hospital mortality rates were significantly higher than the ones in the training set: $51.1 \%$ vs $39.2 \%(P=0.009)$ and $63.7 \%$ vs $54.6 \%$ $(P=0.043)$ respectively. Five $(2.1 \%)$ patients were transplanted during the follow-up and none of these died within the index hospital stay. Median (IQR) length of ICU and hospital stays were 4 (2-9) and 13 (5-25) days respectively. Median (IQR) length of follow-up was 9 (2-19) days.

The overall prognostic performance of the LacOF model for ICU mortality was better in the validation set in comparison to the training set with an AUC of 0.85 vs 0.76 , a $R^{2}$ of 0.32 vs 0.23 and a Brier score of 0.19 vs 0.20 respectively (Table 3 and Figure 1C). Overall, $79 \%$ of the patients were correctly classified. In fact, the LacOF model discriminative ability in the validation set outperformed several other prognostic systems, including the CLIF-SOFA, CLIF-C ACLF, APACHEII and MELD scores (Table 3: $P<0.05$ for all).

The LacOF model calibration in the validation set was good up to the $25 \%$ likelihood of ICU mortality threshold. For higher values in the probability scale, the new predictive model underestimated the likelihood of ICU mortality (Figure 1C). Conversely, the APACHEII score calibration was good for almost the entire probability scale of ICU mortality in the validation set (Figure 1D).

\section{4 | DISCUSSION}

\section{1 | Key findings}

Using a reasonably large international multicentre cohort of patients with ACLF admitted to ICU (training set), we compared the prognostic performance of several scores for ICU mortality. We further derived a new predictive model for ICU mortality using two easy to assess clinical parameters on admission to ICU: lactate and the number of organ failures (based on the CLIF-SOFA score). The

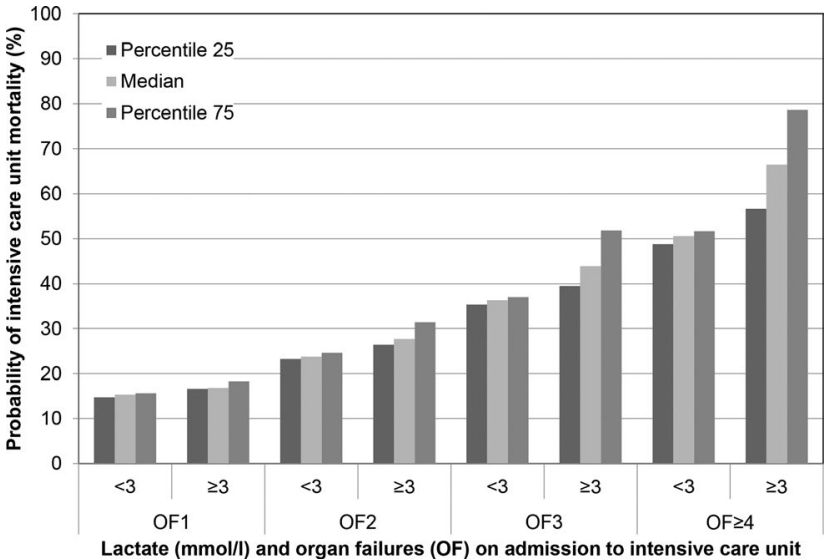

FIGURE 2 Predicted probability of intensive care unit (ICU) mortality based on lactate $(\mathrm{mmol} / \mathrm{L})$ and the number of organ failures on admission to ICU

LacOF model performed well when applied to an external cohort of patients with ACLF admitted to ICU (validation set), outperforming most pre-existent prognostic systems assessed.

\subsection{Comparisons with previous studies}

In our training set, lactate on admission to ICU was significantly associated with ICU mortality: per each $1 \mathrm{mmol} / \mathrm{L}$ increment, an $8 \%$ increased odds of death. Previous studies have suggested that lactate on admission to ICU may have prognostic value for short-term mortality in patients with ACLF.

Using a retrospective cohort of patients with cirrhosis admitted to ICU ( $n=635)$, Theocharidou et al. showed that lactate on admission to ICU was significantly associated with hospital mortality with an aOR of $1.15 .{ }^{14}$ Additionally, Campbell et al. studied a retrospective cohort of 84 critically ill patients with cirrhosis and demonstrated that lactate on admission to ICU was significantly associated with ICU mortality with an aOR of $1.89 .{ }^{15}$ Finally, Edmark et al. assembled 
TAB LE 3 Comparative LacOF model discriminative ability for mortality within the ICU and hospital stays in the training and validation sets

\begin{tabular}{|c|c|c|c|c|}
\hline \multirow[b]{2}{*}{ Model } & \multicolumn{2}{|l|}{ ICU mortality } & \multicolumn{2}{|l|}{ Hospital mortality } \\
\hline & $\operatorname{AUC}(95 \% \mathrm{CI})$ & $P^{a}$ & $\operatorname{AUC}(95 \% \mathrm{Cl})$ & $P^{\mathrm{a}}$ \\
\hline \multicolumn{5}{|l|}{ Training set } \\
\hline LacOF & $0.76(0.70-0.82)$ & NA & $0.77(0.71-0.83)$ & NA \\
\hline CLIF-SOFA & $0.70(0.63-0.77)$ & 0.047 & $0.71(0.64-0.78)$ & 0.014 \\
\hline CLIF-C ACLF & $0.71(0.65-0.76)$ & 0.10 & $0.73(0.67-0.79)$ & 0.08 \\
\hline CLIF-C ACLF Lac & $0.76(0.70-0.82)$ & 0.98 & $0.76(0.70-0.83)$ & 0.62 \\
\hline APACHEII & $0.73(0.66-0.81)$ & 0.61 & $0.71(0.64-0.79)$ & 0.036 \\
\hline MELD & $0.70(0.64-0.77)$ & 0.13 & $0.73(0.66-0.79)$ & 0.21 \\
\hline \multicolumn{5}{|l|}{ Validation set } \\
\hline LacOF & $0.85(0.80-0.90)$ & NA & $0.84(0.78-0.89)$ & NA \\
\hline CLIF-SOFA & $0.79(0.73-0.84)$ & 0.042 & $0.81(0.76-0.86)$ & 0.54 \\
\hline CLIF-C ACLF & $0.73(0.67-0.79)$ & 0.004 & $0.76(0.70-0.81)$ & 0.04 \\
\hline CLIF-C ACLF Lac & $0.82(0.77-0.88)$ & 0.17 & $0.83(0.78-0.89)$ & 0.73 \\
\hline APACHEII & $0.74(0.68-0.79)$ & 0.003 & $0.71(0.64-0.76)$ & 0.001 \\
\hline MELD & $0.78(0.72-0.83)$ & 0.034 & $0.83(0.78-0.88)$ & 0.42 \\
\hline
\end{tabular}

$\mathrm{ICU}$, intensive care unit; $\mathrm{AUC}$, area under the receiver-operating characteristic curve; $\mathrm{Cl}$, confidence interval; LacOF, lactate and number of organ failures model; NA, not applicable; CLIF-SOFA, Chronic Liver Failure - Sequential Organ Failure Assessment score; CLIF-C ACLF, Chronic Liver Failure Acute-on-Chronic Liver Failure score; CLIF-C ACLF Lac, Chronic Liver Failure - Acute-on-Chronic Liver Failure - Lactate score; APACHEII, Acute Physiology and Chronic Health Evaluation II score; MELD, Model for End-stage Liver Disease score.

${ }^{a}$ De Long test for comparing LacOF vs other. a retrospective cohort of patients with cirrhosis admitted to ICU $(n=945)$ and reported that lactate was significantly associated with hospital mortality with an aOR of 1.78 to 4.46 (categorical variable). ${ }^{16}$

For general critically ill patients, lactate has been widely used as a marker of disease severity and associated with higher overall mortality. ${ }^{17,18}$ Biologically, lactate has been accepted as a surrogate of physiological stress, microcirculatory dysfunction or tissue hypoxia from multiple aetiologies. ${ }^{19,20}$ In liver failure, lactate clearance may be further impaired by mechanisms yet to be fully understood. ${ }^{21-23}$ Therefore, to use lactate, an easily accessible and widely used parameter in the ICU setting, as an early prognostic marker in patients with ACLF may be worthwhile.

In our training set, the number of organ failures on admission to ICU (according to the CLIF-SOFA score) was significantly associated with ICU mortality: per each additional organ failure (ranging from 1 to 6), a 77\% increased odds of death. Both in general critically ill patients and in patients with cirrhosis admitted to ICU, previous studies have shown that the number of organ failures that develop during the hospital stay negatively impacts patients' prognosis.

Using a prospective cohort of critically ill patients $(n=1449)$, Vincent et al. showed that the increasing number of organ failures and severity of each organ failure during ICU stay was significantly associated with higher ICU mortality. ${ }^{24}$ Cholongitas et al. used a retrospective cohort of patients with cirrhosis admitted to ICU $(n=312)$ and demonstrated that the number of organ failures on admission to ICU was significantly associated with 6-week mortality with an aOR of 17.6 to 99.1 (categorical variable). ${ }^{25}$
While the number of organ failures present on admission to ICU seems to be important for patients' short-term prognosis, the type of organs failing may impact differently the overall severity of disease. In our training set, all types of organ failures, except for cerebral failure (68.1\% vs $62.3 \%$ ), were univariately associated with higher ICU mortality. While the grade of HE (West Haven criteria) is a well established severity marker of cirrhosis, if cerebral failure develops without other organ failures, it may not be associated with higher ICU mortality. Amongst 22 patients in the training set with cerebral failure (14.2\% of subtotal) without any other organ failure on admission to ICU, only one died during the ICU stay (OR [95\% confidence interval] = 0.06 [0.01-0.48]). This may help explain why cerebral failure was not associated with ICU mortality in our training set. Furthermore, this rationale resembles the one from the CLIF Consortium' definitions of ACLF: patients with single cerebral failure are not considered to have ACLF unless they present a serum creatinine of at least 1.5-1.9 $\mathrm{mg} / \mathrm{dL}^{2}$

While the severity of disease on admission to ICU given by median APACHEII score was similar between the training and validation sets (23 vs $23 ; P=0.77$ ), ICU mortality was significantly higher in the validation set $(39.2 \%$ vs $51.1 \%$; $P=0.009)$. Furthermore, while the APACHEII discriminative ability for the training and validation sets was similar (AUC of 0.73 vs 0.74 ), its calibration was poor for the training set (Figure 1B) but good for the validation set (Figure 1D).

As the validation set was an older cohort than the training set, this difference in the APACHEII calibration was likely because of 
TAB LE 4 Baseline characteristics in the validation set

\begin{tabular}{|c|c|}
\hline & Overall $(n=237)$ \\
\hline Age & $56(49-62)$ \\
\hline Sex (male) & $156(65.8 \%)$ \\
\hline \multicolumn{2}{|l|}{ Aetiology } \\
\hline Alcohol & $83(35.0 \%)$ \\
\hline $\mathrm{HCV}$ & $57(24.1 \%)$ \\
\hline Alcohol + HCV & 35 (14.8\%) \\
\hline Other & $62(26.2 \%)$ \\
\hline $\mathrm{HCC}$ & 34 (14.3\%) \\
\hline Ascites & $182(76.8 \%)$ \\
\hline \multicolumn{2}{|l|}{ Precipitant event } \\
\hline Sepsis & $63(26.6 \%)$ \\
\hline Bleeding & $23(9.7 \%)$ \\
\hline Other & $151(63.7 \%)$ \\
\hline Unknown & 0 \\
\hline \multicolumn{2}{|l|}{ Laboratory } \\
\hline Haemoglobin (g/L) & $82(73-91)$ \\
\hline Leucocytes $\left(10^{3} / \mu \mathrm{L}\right)$ & $12.7(8.4-18.2)$ \\
\hline Platelets $\left(10^{3} / \mu \mathrm{L}\right)(\mathrm{n}=236)$ & $60(40-86)$ \\
\hline INR & $1.8(1.5-2.2)$ \\
\hline Bilirubin (mg/dL) & $6.4(2.8-13.2)$ \\
\hline Albumin (g/dL) (n = 232) & $23.0(19.0-28.0)$ \\
\hline Creatinine (mg/dL) & $1.91(1.12-3.09)$ \\
\hline Sodium (mmol/L) & $141(133-145)$ \\
\hline Lactate $(\mathrm{mmol} / \mathrm{L})(\mathrm{n}=213)$ & $4.7(2.3-10.0)$ \\
\hline Organ failures ${ }^{a}$ & $2(2-3)$ \\
\hline Liver & 69 (29.1\%) \\
\hline Kidneys & $122(51.5 \%)$ \\
\hline Brain & $67(28.3 \%)$ \\
\hline Coagulation & $51(21.5 \%)$ \\
\hline Circulation & $130(54.9 \%)$ \\
\hline Lungs & $164(69.2 \%)$ \\
\hline \multicolumn{2}{|l|}{ Organ support } \\
\hline RRT & 43 (18.1\%) \\
\hline Vasopressors & $130(54.9 \%)$ \\
\hline IMV & $214(90.3 \%)$ \\
\hline PF ratio $(\mathrm{mmHg})$ & $145(98-220)$ \\
\hline \multicolumn{2}{|l|}{ ACLF grade ${ }^{a}$} \\
\hline No ACLF & $6(2.5 \%)$ \\
\hline 1 & 46 (19.4\%) \\
\hline 2 & 74 (31.2\%) \\
\hline 3 & 111 (46.8\%) \\
\hline CLIF-SOFA & $14(12-16)$ \\
\hline CLIF-C ACLF & $58(51-65)$ \\
\hline CLIF-C ACLF Lac & $62(54-75)$ \\
\hline APACHEII & $23(19-28)$ \\
\hline
\end{tabular}

TA B L E 4 (Continued)

\begin{tabular}{ll} 
& Overall $(\mathbf{n}=237)$ \\
\hline MELD & $27(20-35)$ \\
\hline
\end{tabular}

ICU, intensive care unit; $\mathrm{HCV}$, hepatitis $\mathrm{C}$ virus; $\mathrm{HCC}$, hepatocellular carcinoma; INR, international normalized ratio; RRT, renal replacement therapy; IMV, tracheal intubation with or without invasive mechanical ventilation; $\mathrm{PF}$ ratio, arterial oxygen partial pressure and oxygen inspiration fraction ratio; ACLF, acute-on-chronic liver failure; CLIF-SOFA, Chronic Liver Failure - Sequential Organ Failure Assessment score; CLIF-C ACLF, Chronic Liver Failure - Acute-on-Chronic Liver Failure score; CLIF-C ACLF Lac, Chronic Liver Failure - Acute-on-Chronic Liver Failure - Lactate score; APACHEII, Acute Physiology and Chronic Health Evaluation II score; MELD, Model for End-stage Liver Disease score.

${ }^{a}$ Definitions of organ failures based on CLIF-SOFA score.

the expected decrease in ICU mortality from the 2000-2011 period (validation set) to the 2010-2016 period (training set). Therefore, this ICU mortality discrepancy between the two sets was likely related to the overall improvements in critical care medicine over the past decades, including the management of patients with cirrhosis. In fact, McPhail et al. used a retrospective cohort of patients with cirrhosis admitted to ICU $(n=971)$ to show that hospital survival has significantly improved from $40 \%$ in 2000 to $63 \%$ in 2010 $(P<0.001){ }^{6}$ Furthermore, this emphasizes that using the APACHEII score to prognosticate the outcomes of patients with ACLF currently may be less accurate than decades ago.

Despite this limitation of the validation set, the LacOF model overall prognostic performance was good in a substantially different external validation set ( $R^{2}$ of 0.32 and Brier score of 0.19$)$. While its discriminative ability outperformed most other prognostic systems analysed (AUC of 0.85), its calibration was good up to the $25 \%$ likelihood of ICU mortality threshold, following which it started to underestimate that probability. This pattern of miscalibration may have been tied also to the higher ICU mortality rate in the validation set.

Drolz et al. recently derived the CLIF-C ACLF Lac score to prognosticate 28-day mortality following admission to ICU in patients with cirrhosis. ${ }^{10}$ However, they studied the prognostic performance of their model based on discriminative ability only (AUC of 0.79 ). ${ }^{10}$ While the LacOF and CLIF-C ACLF Lac scores had similar discriminative ability in our validation set (AUC of 0.85 vs 0.82 ), we were not able to compare these models based on the calibration. By assessing the prognostic performance of the LacOF model with both discriminative ability and calibration, we hope to have strengthened our model's overall predictive ability. Therefore, the LacOF model (two variables) may be a simpler and still reliable alternative tool for predicting individual outcomes of patients with cirrhosis and organ failures admitted to ICU, even in comparison with the CLIF-C ACLF Lac score (four variables). ${ }^{10}$

\section{3 | Limitations}

This study has the following limitations to be considered. Firstly, this was a retrospective study, thus may have been prone to 
selection bias. However, the international multicentre character, the reasonably large sample size (similar to studies previously reporting on patients with $\mathrm{ACLF}$ in $\mathrm{ICU}^{2,7}$ ), the objective inclusion and exclusion criteria, and the low level of missing data sure have helped to mitigate the likelihood of such bias. Secondly, as implied previously, the older character of the external validation set may have precluded a more definitive assessment of the LacOF model external validity. However, that comparative analysis of several scoring systems using a substantially different external sample still retains added value. ${ }^{26}$ Definitively, further studies are needed to externally validate the LacOF model predictive ability using preferentially recent samples of patients with cirrhosis and organ failures admitted to ICU.

Despite these limitations, we feel that the results of our study are methodologically sound and clinically relevant. We hope to have added to the previous literature on the management of patients with ACLF. Furthermore, we hope to have helped both intensivists and hepatologists to better prognosticate the individual outcomes of patients with ACLF in the ICU setting, thus contributing to help them improve decisions on both timing and level of organ support in a frequently difficult clinical context.

\section{5 | CONCLUSIONS}

In patients with ACLF, lactate and the number of organ failures on admission to ICU are useful to predict ICU mortality. This early prognostic evaluation may present an alternative to older prognostic tools in use in the ICU setting, for example the APACHEII score, and help clinicians to better stratify the likelihood of ICU mortality and thus optimize organ support strategies.

\section{CONFLICT OF INTEREST}

None to declare.

\section{AUTHORS' CONTRIBUTIONS}

FSC conceived the idea of this study, collected data, performed statistical analysis and drafted the final manuscript. JGA performed statistical analysis and helped to draft the final manuscript. ES, JJR, LB and MJM collected data and critically revised the final manuscript. CJK conceived the idea of this study, collected data and helped to draft the final manuscript.

\section{ORCID}

Filipe S. Cardoso (iD https://orcid.org/0000-0002-6221-6435

Juan G. Abraldes (iD https://orcid.org/0000-0003-3421-937X

Mark J. Mcphail (iD https://orcid.org/0000-0001-5449-2424

Constantine J. Karvellas (iD https://orcid.

org/0000-0002-1555-1089

\section{REFERENCES}

1. Olson JC, Kamath PS. Acute-on-chronic liver failure: concept, natural history, and prognosis. Curr Opin Crit Care. 2011;17:165-169.

2. Moreau R, Jalan R, Gines P, et al. Acute-on-chronic liver failure is a distinct syndrome that develops in patients with acute decompensation of cirrhosis. Gastroenterology. 2013;144:1426-1437.

3. Gustot T, Fernandez J, Garcia E, et al. Clinical Course of acute-onchronic liver failure syndrome and effects on prognosis. Hepatology. 2015;62:243-252.

4. Karvellas CJ, Bagshaw SM. Advances in management and prognostication in critically ill cirrhotic patients. Curr Opin Crit Care. 2014;20:210-217.

5. Artru F, Louvet A, Ruiz I, et al. Liver transplantation in the most severely ill cirrhotic patients: a multicenter study in acute-on-chronic liver failure grade 3. J Hepatol. 2017;67:708-715.

6. McPhail MJ, Shawcross DL, Abeles RD, et al. Increased survival for patients with cirrhosis and organ failure in liver intensive care and validation of the chronic liver failure-sequential organ failure scoring system. Clin Gastroenterol Hepatol. 2015;13:1353-1360.

7. Jalan R, Saliba F, Pavesi M, et al. Development and validation of a prognostic score to predict mortality in patients with acute-onchronic liver failure. J Hepatol. 2014;61:1038-1047.

8. World Medical Association. World Medical Association Declaration of Helsinki: ethical principles for medical research involving human subjects. JAMA. 2013;310:2191-2194.

9. von Elm E, Altman DG, Egger M, Pocock SJ, Gøtzsche PC, Vandenbroucke JP; STROBE Initiative. Strengthening the Reporting of Observational Studies in Epidemiology (STROBE) statement: guidelines for reporting observational studies. BMJ. 2007;335:806-808.

10. Drolz A, Horvatits T, Rutter K, et al. Lactate improves prediction of short-term mortality in critically ill patients with cirrhosis: a multinational study. Hepatology. 2019;69:258-269.

11. Knaus WA, Draper EA, Wagner DP, Zimmerman JE. APACHE II: a severity of disease classification system. Crit Care Med. 1985;13:818-829.

12. Malinchoc M, Kamath PS, Gordon FD, Peine CJ, Rank J, ter Borg PC. A model to predict poor survival in patients undergoing transjugular intrahepatic portosystemic shunts. Hepatology. 2000;31:864-871.

13. Models for Prediction. Clinical prediction models. In: Steyerberg EW, ed. A Practical Approach to Development, Validation, and Updating. New York: Springer; 2009:53-83.

14. Theocharidou E, Pieri G, Mohammad AO, et al., et al. Hospital score: a calibrated prognostic model for patients with cirrhosis admitted to intensive care unit. Comparison with current models and CLIFSOFA score. Am J Gastroenterol. 2014;109:554-562.

15. Campbell J, McPeake J, Shaw M, et al. Validation and analysis of prognostic scoring systems for critically ill patients with cirrhosis admitted to ICU. Crit Care. 2015;19:364.

16. Edmark C, McPhail MJ, Bell M, Whitehouse T, Wendon J, Christopher KB. LiFe: a liver injury score to predict outcome in critically ill patients. Intensive Care Med. 2016;42:361-369.

17. Masevicius FD, Rubatto Birri PN, Risso Vazquez A, et al. Relationship of at admission lactate, unmeasured anions, and chloride to the outcome of critically ill patients. Crit Care Med. 2017;45:e1233-e1239. https://doi.org/10.1097/CCM.0000000000002730.

18. Zhang Z, Xu X. Lactate clearance is a useful biomarker for the prediction of all-cause mortality in critically ill patients: a systematic review and meta-analysis*. Crit Care Med. 2014;42:2118-2125.

19. Vellinga NAR, Boerma EC, Koopmans M, et al. Mildly elevated lactate levels are associated with microcirculatory flow abnormalities and increased mortality: a microSOAP post hoc analysis. Crit Care. 2017;21:255 
20. Bakker J, Nijsten MWN, Jansen TC. Clinical use of lactate monitoring in critically ill patients. Ann Intensive Care. 2013;3:12.

21. Jeppesen JB, Mortensen C, Bendtsen F, Møller S. Lactate metabolism in chronic liver disease. Scand J Clin Lab Invest. 2013;73:293-299.

22. Freire Jorge $\mathrm{P}$, Wieringa $\mathrm{N}$, de Felice $\mathrm{E}$, van der Horst ICC, Oude Lansink A, Nijsten MW. The association of early combined lactate and glucose levels with subsequent renal and liver dysfunction and hospital mortality in critically ill patients. Crit Care. 2017;21:218.

23. Sterling SA, Puskarich MA, Jones AE. The effect of liver disease on lactate normalization in severe sepsis and septic shock: a cohort study. Clin Exp Emerg Med. 2015;2:197-202.

24. Vincent JL, Moreno R, Takala J, et al. The SOFA (Sepsis-related Organ Failure Assessment) score to describe organ dysfunction/ failure. On behalf of the Working Group on Sepsis-Related Problems of the European Society of Intensive Care Medicine. Intensive Care Med. 1996;22:707-710.

25. Cholongitas E, Senzolo M, Patch D, et al. Risk factors, sequential organ failure assessment and model for end-stage liver disease scores for predicting short term mortality in cirrhotic patients admitted to intensive care unit. Aliment Pharmacol Ther. 2006;23:883-893.
26. Reverter E, Tandon P, Augustin S, et al. A MELD-based model to determine risk of mortality among patients with acute variceal bleeding. Gastroenterology. 2014;146:412-419.

\section{SUPPORTING INFORMATION}

Additional supporting information may be found online in the Supporting Information section at the end of the article.

How to cite this article: Cardoso FS, Abraldes JG, Sy E, et al. Lactate and number of organ failures predict intensive care unit mortality in patients with acute-on-chronic liver failure. Liver Int. 2019;39:1271-1280. https://doi.org/10.1111/ liv. 14083 